\title{
Terephthalic Acid Synthesis at Higher Concentrations in High-Temperature Liquid Water. 1. Effect of Oxygen Feed Method
}

\author{
Mitsumasa Osada \\ Dept. of Chemical Engineering, Ichinoseki National College of Technology, Takanashi, Hagisho, Ichinoseki, \\ Iwate 021-8511, Japan \\ Phillip E. Savage \\ Dept. of Chemical Engineering, University of Michigan, Ann Arbor, MI 48109
}

DOI 10.1002/aic.11718

Published online February 4, 2009 in Wiley InterScience (www.interscience.wiley.com).

\begin{abstract}
We conducted terephthalic acid (TPA) synthesis from p-xylene in high-temperature liquid water $(H T W)$ at $300^{\circ} \mathrm{C}$. The p-xylene concentration at the reaction condition was 0.2 mol $L^{-1}$, which is the highest to date in research that achieved at least 80 mol \% yields of TPA in HTW. Pure oxygen gas was the oxidant. Increasing the $\mathrm{MnBr}_{2}$ catalyst concentration increased the rate of TPA formation only slightly. In contrast, whether oxygen was fed in small, quick, discrete bursts, or fed continuously significantly affected the p-xylene conversion and the TPA selectivity. Adding oxygen in quick bursts and small increments led to high selectivities (>90 mol \%) of TPA. Continuous addition of oxygen failed to do so. In addition to identifying the sensitivity of this synthesis to the oxygen feed method, these results also demonstrate the feasibility of HTW for TPA synthesis at higher concentrations, and hence high TPA production per unit reactor volume. (C) 2009 American Institute of Chemical Engineers AIChE J, 55: 710716, 2009

Keywords: environmental engineering, supercritical processes, catalysis
\end{abstract}

\section{Introduction}

High-temperature liquid water (HTW) as a reaction medium is attractive for organic chemical synthesis because water is inexpensive, abundant, nontoxic, environmentally benign and has desirable chemical properties. ${ }^{1,2}$ Using water as a substitute for organic solvents is consistent with the principles of Green Chemistry. The properties of HTW including a decreased dielectric constant and elevated ion product (relative to ambient water) are among the underlying

Correspondence concerning this article should be addressed to P. E. Savage at psavage@umich.edu.

(C) 2009 American Institute of Chemical Engineers causes of its successful application as a solvent for organic reactions. HTW affords some solubility for small organic compounds that are largely insoluble in ambient water.

A particularly suitable large-scale industrial reaction system for a HTW process is $p$-xylene oxidation to terephthalic acid (TPA). ${ }^{3,4}$ TPA is a major commodity chemical and an important intermediate for the production of polyester. It is produced commercially by homogeneously catalyzed selective oxidation of $p$-xylene in acetic acid and then purified by hydrogenation in high-temperature water. There are several advantages attendant to using HTW as the reaction medium for this process. First, the use of acetic acid necessitates an expensive distillation step to separate the water formed during the oxidation reaction from the acetic acid so that it can 
be recycled back to the reactor. Additionally, acetic acid is oxidized during the $p$-xylene oxidation reaction and these losses must be offset by replenishing the solvent. Finally, acetic acid reacts with the bromide catalysts used commercially to make methyl bromide, a hazardous compound that must be removed from the gaseous effluent stream.

The technical feasibility of a HTW process for TPA synthesis has been demonstrated. ${ }^{5-12}$ Our group has reported on p-xylene partial oxidation in HTW and found that the highest TPA yields $(>80 \mathrm{~mol} \%)$ were obtained at $300^{\circ} \mathrm{C}$, [p-xylene $]_{0}=0.02 \mathrm{~mol} \mathrm{~L}{ }^{-1},\left[\mathrm{O}_{2}\right]_{0}=0.10 \mathrm{~mol} \mathrm{~L}^{-1},\left[\mathrm{MnBr}_{2}\right]=$ $0.007 \mathrm{~mol} \mathrm{~L}^{-1}$, and $t=5-15 \mathrm{~min} .{ }^{5}$ Moreover, under these conditions and $t=15 \mathrm{~min}$, the yields of carbon monoxide and carbon dioxide, which are products from burning, were below $2 \% . \mathrm{MnBr}_{2}$ was the most active catalyst of those we assessed. Hamley et al. ${ }^{9}$ conducted $p$-xylene partial oxidation in supercritical water at $400^{\circ} \mathrm{C}$ and $[p \text {-xylene }]_{0}=0.008-0.05$ mol $\mathrm{L}^{-1}$ by using a flow reactor and reported TPA yields exceeding $90 \%$ at residence time less than $1 \mathrm{~min}$.

Although HTW has been demonstrated to be an effective medium for TPA synthesis, work to date that produced high TPA yields has been done with low $p$-xylene loadings. These low $p$-xylene concentrations (below $0.05 \mathrm{~mol} \mathrm{~L}^{-1}$ ) naturally lead to low productivity of TPA per unit reactor volume. It would be advantageous to operate with higher $p$-xylene loadings so more TPA could be made per unit reactor volume. The issue then becomes whether high TPA yields and low byproduct yields still could be achieved from synthesis in HTW.

In this work, we conducted synthesis at a higher concentration $\left([p \text {-xylene }]_{0}=0.2 \mathrm{~mol} \mathrm{~L}^{-1}\right)$ in $\mathrm{HTW}$ at $300^{\circ} \mathrm{C}$. The $p$-xylene concentration in this work is 10 times that of our previous work, which used the same stirred batch reactor system and provided high TPA yields. ${ }^{5}$ Another key difference from our previous work is that pure oxygen gas was selected as the oxidant and it was added into the batch reactor by a syringe pump. We also altered the manner in which $p$-xylene and catalyst were introduced into the reactor. These changes were required because of the 10 -fold higher $p$-xylene concentration used in this study. If we did not make these operational changes, the reactor pressure would have been 10 times higher than it was in our previous work and exceeded the safe operating region of the autoclave.

In this article, we report new information concerning the effect of the $\mathrm{MnBr}_{2}$ catalyst concentration and the oxygen feed method on the partial oxidation of $p$-xylene at higher concentrations in HTW. In particular, we show that the manner in which oxygen is added to the reactor has a profound influence on the TPA yield and selectivity.

\section{Experimental Section}

We purchased all chemicals from Sigma-Aldrich in high purity and used them as received. All experiments were conducted in a $440 \mathrm{~mL}$ Hastelloy FC series autoclave batch reactor fabricated by PPI (Warminster, PA). The reactor is equipped with a stirrer, dip tube for sampling, and a separate tube for addition of oxygen gas. This latter tube was connected to a syringe pump (Isco model 260D), which enables us to control and alter how oxygen gas is added into the preheated HTW reaction medium. The reactor has instrumentation for measuring temperature and pressure.

An experiment began with loading distilled, deionized water $(270 \mathrm{~mL}$ at room temperature $), p$-xylene $(10 \mathrm{~mL})$, and carefully measured amounts of $\mathrm{MnBr}_{2}$ catalyst into the reactor. We added enough water and $p$-xylene such that the liquid phase occupied $\sim 90 \%$ of the reactor volume at the reaction temperature of $300^{\circ} \mathrm{C}$. Therefore, we expect two separate liquid and vapor phases to be present in the reactor at reaction conditions. $p$-Xylene solubility in HTW at $294.9^{\circ} \mathrm{C}$ has been reported as 0.0448 ( $\mathrm{g} p$-xylene/g water), which is above the concentration of 0.0319 (g $p$-xylene/g water) used in this work. ${ }^{13}$ Therefore, a single liquid phase should exist in the reactor at reaction conditions. After the reactor had been loaded with water, $p$-xylene, and $\mathrm{MnBr}_{2}$ catalyst, it was sealed by bolting the base to the head.

Next, we purged the batch reactor with nitrogen and began heating and stirring (at $840 \mathrm{rpm}$ ). When the reactor reached the set point temperature, we withdrew samples from the liquid phase into stainless steel sample bulbs, which had a nominal volume of $0.8 \mathrm{~mL}$, to check the prereaction $p$-xylene selectivity. Pure oxygen from a gas cylinder was fed to the syringe pump and then a valve between the gas cylinder and the syringe pump was closed. We measured the moles of oxygen by the syringe pump internal volume and pressure. The oxygen in the syringe pump was taken to a pressure exceeding that in the reactor. Then, we opened a valve between the syringe pump and the reactor and ran the syringe pump. The introduction of oxygen gas into the reactor defined $t=0$ for the reaction. We added oxygen gas several times throughout the reaction, controlling the amount added and speed of addition by programming the syringe pump. Oxygen addition was done in this manner for safety purposes. We desired to avoid reactor conditions with large amounts of both $p$-xylene and oxygen present simultaneously, and we also desired to keep the system pressure well below the rated pressure of the autoclave. As the reaction progressed, we withdrew samples from the liquid phase into the sample bulbs. We purged completely any residual material in the transfer line between the dip tube and the sample bulb immediately before collecting the sample for analysis. Weighing each sample bulb before and after sampling provides the mass of the sample collected. The sample bulbs were placed in a refrigerator for $24 \mathrm{~h}$ to minimize the loss of any volatile products upon bulb opening.

After the reaction, the batch reactor was cooled to ambient temperature. We then opened the reactor and recovered water and water-soluble products first. After which the remaining liquid and solid products were recovered by using about 100-300 mL of dimethyl sulfoxide (DMSO) solvent. We also recovered the products in the sample bulbs. We rinsed the bulb several times with DMSO and combined all liquid recovered from the bulb in a $10 \mathrm{~mL}$ volumetric flask. We analyzed both the products in the batch reactor after the reaction and those in the sample bulbs via HPLC using a method described previously. ${ }^{6}$ This HPLC analysis provides the concentration of each compound, which then admits calculation of product selectivity and yield. The volume of the sample bulb plus material purged during sampling $(3.2 \mathrm{~mL})$ was small in comparison with that of the batch reactor $(440 \mathrm{~mL})$, 
Table 1. Product Selectivities and Yields in the Autoclave Reactor After Reaction*

\begin{tabular}{|c|c|c|c|c|c|c|c|c|c|c|}
\hline \multirow[b]{2}{*}{ Run } & \multicolumn{3}{|c|}{ Conditions } & \multirow[b]{2}{*}{$\begin{array}{c}\text { Aromatic } \\
\text { Ring Balance } \\
(\mathrm{mol} \mathrm{\%})\end{array}$} & \multicolumn{6}{|c|}{ Selectivity (mol \%) (Product Yield (mol \%)) } \\
\hline & $\mathrm{O}_{2}$ step $^{\dagger}$ & $\begin{array}{c}\text { Final } \\
\mathrm{O}_{2} / p \text {-xylene } \\
(\mathrm{mol} / \mathrm{mol})\end{array}$ & $\begin{array}{c}\mathrm{MnBr}_{2} \\
\left(\mathrm{~mol} \mathrm{~L}^{-1}\right)\end{array}$ & & TPA & $p$-Xylene & $\begin{array}{l}p \text {-Tolu } \\
\text { aldehyde }\end{array}$ & $\begin{array}{c}p \text {-Toluic } \\
\text { acid }\end{array}$ & 4-CBA & $\mathrm{BA}$ \\
\hline 1 & 0.5 & 9 & 0.014 & 92.8 & $97.6(90.0)$ & $0.0(0.0)$ & $0.2(0.2)$ & $0.1(0.1)$ & $0.0(0.0)$ & $2.1(1.9)$ \\
\hline 2 & 0.5 & 9 & 0.028 & 74.8 & $94.7(67.6)$ & $0.0(0.0)$ & $0.7(0.5)$ & $0.7(0.5)$ & $0.4(0.3)$ & $3.5(2.5)$ \\
\hline 3 & 1.0 & 9 & 0.028 & 87.3 & $96.1(83.0)$ & $0.0(0.0)$ & $0.8(0.7)$ & $1.4(1.2)$ & $0.1(0.1)$ & $1.6(1.4)$ \\
\hline 4 & cont $^{+}$ & 6 & 0.028 & 97.2 & $7.3(7.1)$ & $87.9(85.2)$ & $1.4(1.3)$ & $3.5(3.4)$ & $0.0(0.0)$ & $0.0(0.0)$ \\
\hline 5 & cont $^{+}$ & 6 & 0.028 & 77.3 & $31.4(23.8)$ & $59.7(45.3)$ & $5.4(4.1)$ & $3.5(2.6)$ & $0.0(0.0)$ & $0.0(0.0)$ \\
\hline 6 & cont $^{+}$ & 6 & 0.028 & 81.6 & $31.1(25.0)$ & $59.4(47.7)$ & $5.4(4.3)$ & $3.4(2.8)$ & $0.7(0.6)$ & $0.0(0.0)$ \\
\hline 7 & cont $^{+}$ & 6 & 0.028 & 75.8 & $31.8(23.6)$ & $65.6(48.5)$ & $1.0(0.8)$ & $1.0(0.8)$ & $0.0(0.0)$ & $0.6(0.5)$ \\
\hline
\end{tabular}

* Reaction condition; $p$-xylene $0.2 \mathrm{~mol} \mathrm{~L}^{-1}$.

${ }^{\dagger}$ The molar ratio of $\mathrm{O}_{2}$ added to $p$-xylene initially loaded. The stoichiometric amount needed to convert $p$-xylene to TPA is 3 .

${ }^{*}$ cont $=$ continuous addition of $\mathrm{O}_{2}$.

so $\sim 90 \mathrm{~mol} \%$ of the initial material added to the reactor should remain within it at the end.
The aromatic ring balance, selectivity, and product yield are defined as follows:

$$
\begin{aligned}
& \text { aromatic ring balance }(\mathrm{mol} \%)=\frac{\begin{array}{c}
\text { sum of moles of aromatic compounds recovered from } \\
\text { the autoclave and from the sample bulbs }
\end{array}}{\text { moles of } p \text {-xylene loaded into the autoclave }} \times 100 \\
& \text { selectivity }(\mathrm{mol} \%)=\frac{\text { moles of product } i \text { (in autoclave or sample bulb) }}{\text { sum of moles of all organic products (in autoclave or sample bulb) }} \times 100 \\
& \text { product yield }(\mathrm{mol} \%)=\frac{\text { moles of product } i \text { recovered from autoclave after reaction }}{\text { moles } p \text {-xylene loaded in autoclave-moles aromatic compounds in sample bulbs }} \times 100
\end{aligned}
$$

\section{Results}

Table 1 provides a summary of the experimental conditions and the results based upon the material recovered postreaction from the autoclave reactor. We selected experimental conditions based on our previous research that had achieved high TPA yields (>80 mol \%). For examlpe, Run 1 used 10 times the $p$-xylene loading and two times the $\mathrm{MnBr}_{2}$ loading of a previous experiment at lower $p$-xylene concentrations. ${ }^{5}$ All runs were done at a nominal reaction temperature of $300^{\circ} \mathrm{C}$ and with pure oxygen as oxidant. As described below in detail, the reaction time of each run is different and is from 50 to $150 \mathrm{~min}$. Table 1 shows that the main products obtained in these experiments were TPA, $p$-tolualdehyde, $p$ toluic acid, 4-carboxybenzaldehyde (4-CBA), and benzoic acid (BA). We also analyzed for 4-methyl benzyl alcohol, 4hydroxymethyl benzoic acid, terephthaldicarboxaldehyde, and phenol, but none of these products were detected in this work. All runs shown in Table 1 were carried out once. The aromatic ring balance for each run was above $74.8 \%$ and in Run 4 it was up to $97.2 \mathrm{~mol} \%$. The loss of aromatic rings is probably due to the formation of gas-phase products (e.g., $\mathrm{CO}, \mathrm{CO}_{2}$ ) and the inability to recover all of the water-insoluble solid products such as TPA. The reactor internals, in particular, had many irregular surfaces, which made it very difficult for the complete recovery of the material that adhered to them. As described below in detail, Run 5 and 6 are almost the same experimental conditions. Since the selectivities of Run 5 and 6 are similar, we think that the error of the selectivities is very small.

Figure 1 displays the temporal variations of the product selectivities obtained from analysis of the sample bulbs, the molar ratio of total oxygen fed to $p$-xylene in the reactor, pressure, and temperature from Run 1. The increment of oxygen feed was 0.5 moles $\mathrm{O}_{2}$ per mole $p$-xylene initially fed, which is 0.04 moles $\mathrm{O}_{2}$ added per increment. The ultimate oxygen $/ p$-xylene ratio in the reactor was 9 . The stoichiometric ratio needed to convert $p$-xylene to TPA is 3 . Figure $1 \mathrm{c}$ shows that the pressure gradually increased with the addition of oxygen. More specifically, the pressure increased at each oxygen injection, and then decreased, presumably because oxygen was being consumed in the partial oxidation of $p$-xylene. When the first injection of oxygen was made, the temperature increased about $10^{\circ} \mathrm{C}$, even though the heater output was fixed throughout the entire experiment. After $t=0$, the temperature was stable at around $(298 \pm 3)^{\circ} \mathrm{C}$.

Figure 1a shows that $p$-xylene disappeared gradually and $p$-tolualdehyde was the product present in the highest selectivity at about $20 \mathrm{~min}$. The $p$-tolualdehyde selectivity then decreased as it was oxidized to $p$-toluic acid, which subsequently formed 4-CBA. The selectivity of $p$-toluic acid and 4-CBA increased to a maximum around $60 \mathrm{~min}$ and then decreased as $p$-toluic acid and 4-CBA were oxidized further to TPA. Benzoic acid was not observed, which indicates 



(c)

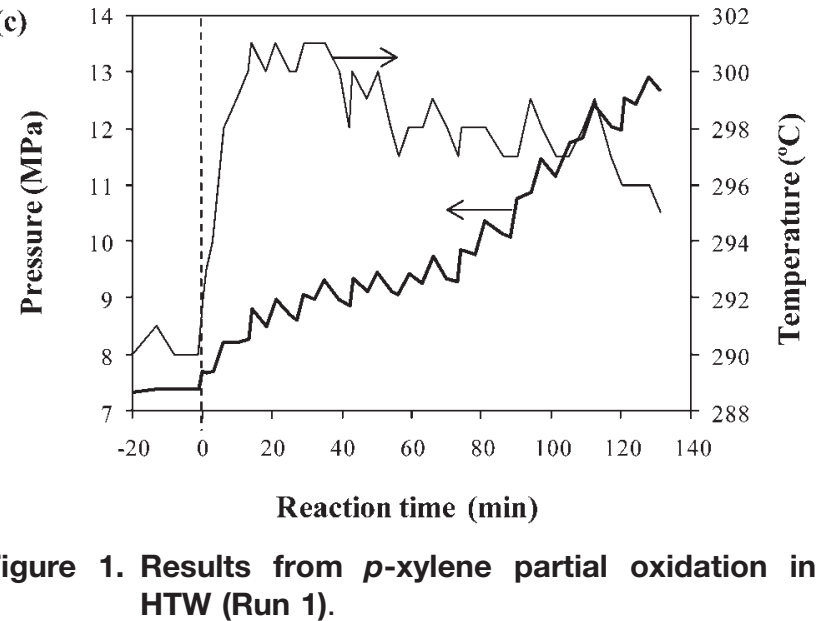

(a) Product selectivity, (b) oxygen feed program, and (c) reactor pressure and temperature.

there was little decarboxylation of TPA occurring. The highest TPA selectivity was $92.8 \mathrm{~mol} \%$.

The product selectivities obtained from analysis of the material recovered from the autoclave after the reaction (shown in Table 1) are comparable to those from the final sample bulbs in Figure 1a. A small amount of benzoic acid was observed in the batch reactor after the reaction. It was probably present here because this material was exposed to high temperature for a longer time than was the material in the sample bulbs. It took about $60 \mathrm{~min}$ for the batch reactor temperature to cool from 300 to $200^{\circ} \mathrm{C}$. The TPA selectivity and yield for Run 1 in Table 1 were $97.6 \mathrm{~mol} \%$ and $90.0 \mathrm{~mol}$ $\%$, respectively, which are as high as those reported previ- ously for experiments in HTW at much lower p-xylene concentrations. $^{5,9}$

To study the effect of catalyst amount at higher $p$-xylene concentration condition, we conducted an experiment (Run 2) with an initial catalyst concentration of $0.028 \mathrm{~mol} \mathrm{~L}^{-1}$, which is twice the catalyst amount of Run 1 . Figure 2 shows the temporal variations of the product selectivity and the oxygen feed for Run 2. The oxygen feed increment was the same as in Run 1 . The $p$-xylene disappeared at about $30 \mathrm{~min}$ as opposed to $50 \mathrm{~min}$ in Run 1. The formation of TPA in Run 2 was slightly faster than that in Run 1. Both of these results indicate that increasing the catalyst concentration increases the reaction rate. This result is consistent with our previous work at lower $p$-xylene concentration conditions. ${ }^{5}$

In Table 1, the postreaction TPA selectivity for Run 2 was $94.7 \mathrm{~mol} \%$, which is in good correspondence with that from the final sample bulbs in Figure 2. The product selectivities in Runs 1 and 2 are almost the same, which indicates that the higher catalyst concentration affected only the reaction rate and not the final product distribution. The aromatic ring balance from Run 2 was lower than that of Run 1. The main reason is probably the difficulty in recovering all of the solid products from the autoclave, but there is also the possibility that the higher catalyst concentration promoted more burning.
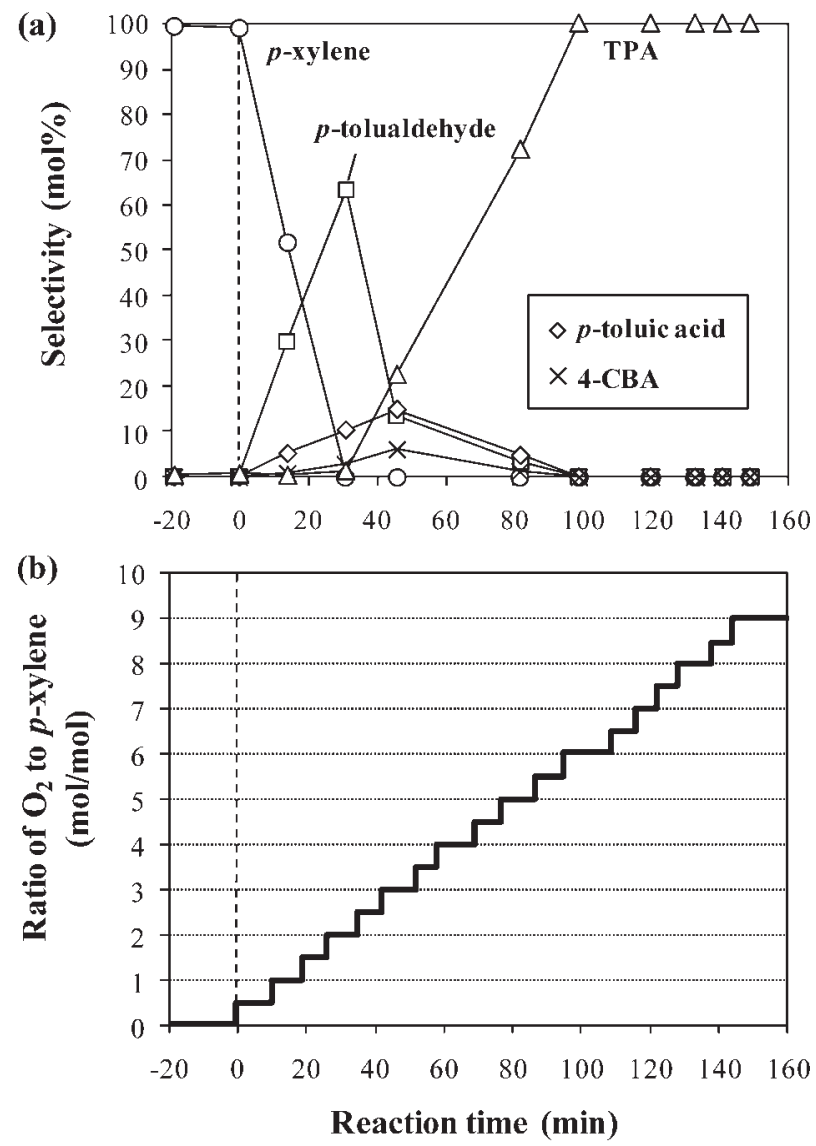

Figure 2. Results from $p$-xylene partial oxidation in HTW (Run 2).

(a) Product selectivity and (b) oxygen feed program. 

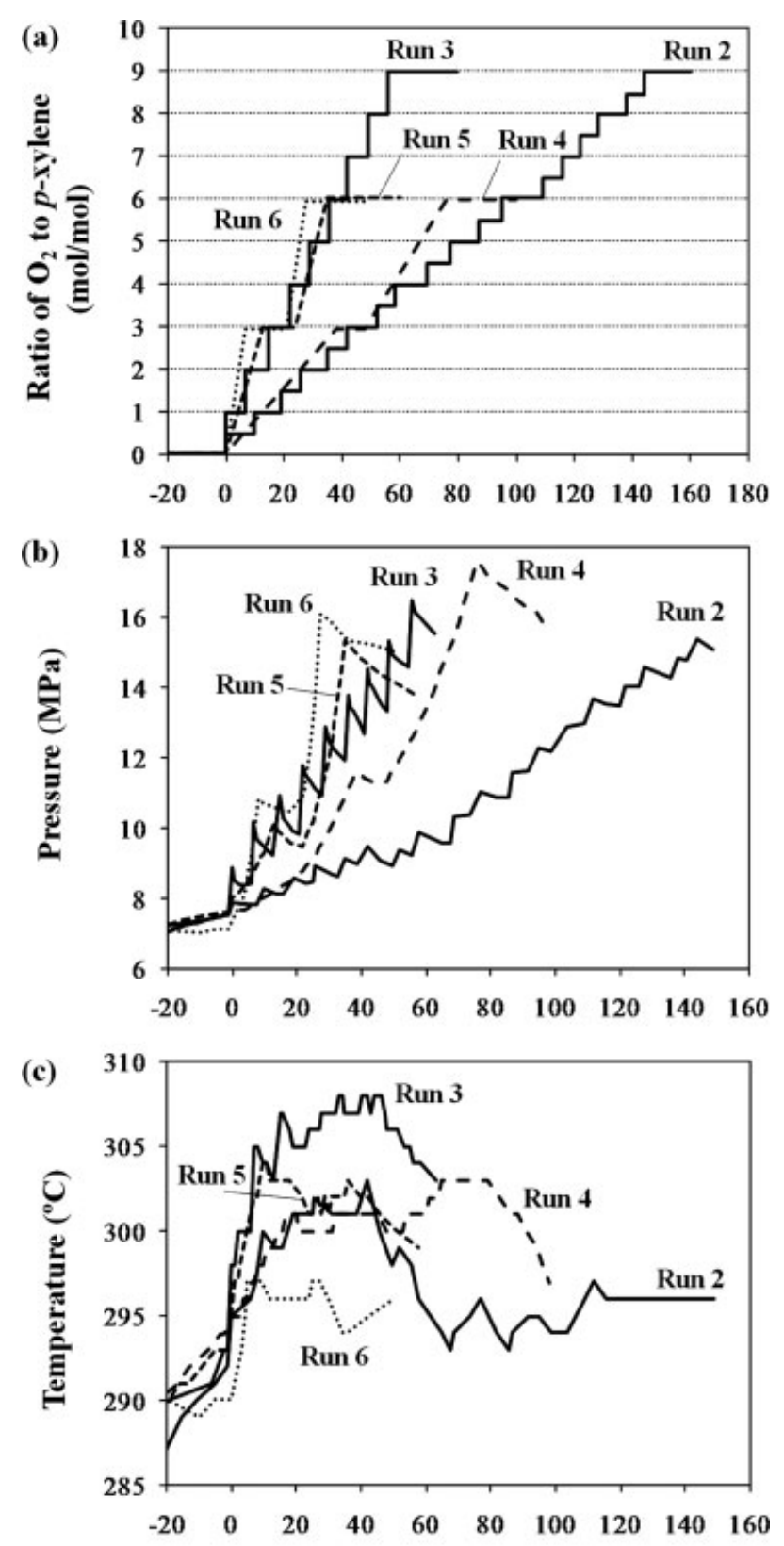

Reaction time (min)

Figure 3. Comparison of reaction conditions for Runs 2-6.

(a) Oxygen feed program, (b) pressure, and (c) temperature.

To assess the effect of the oxygen feed method on the reaction, we conducted experiments (Runs 3-6) under conditions identical to those of Run 2 except for the oxygen feed method. Figure 3 shows the different oxygen feed strategies and resulting reactor pressure and temperature profiles for Runs 2-6. The chief difference in the oxygen feed method employed is that Runs 2 and 3 used discrete incremental addition of oxygen in quick bursts, whereas Runs 4-6 used continuous addition of oxygen.

Figure 4 displays the product selectivity from Run 3 along with the molar ratio of total oxygen fed to $p$-xylene. In Run
3 , the oxygen feed increment is 1.0 mole $\mathrm{O}_{2}$ per mole $p$-xylene initially loaded into the reactor. Since there are fewer oxygen feed increments, the reaction time is shorter than Run 2. p-Xylene disappeared at about $30 \mathrm{~min}$. The formation rate of TPA in Run 3 was significantly faster than that in Run 2, indicating that faster oxygen feed can increase the reaction rate. Table 1 shows that the postreaction TPA selectivity of Run 3 was $96.1 \mathrm{~mol} \%$, which is comparable to the results from the final sample bulbs in Figure 4. The product selectivities from Runs 2 and 3 were almost the same, but the time required was less for Run 3 . These results indicate that the oxygen feed method is a powerful tool for manipulating the rate of $p$-xylene partial oxidation in HTW.

In Run 4, oxygen was fed continuously in two discrete ramps. Recall that step-wise addition of oxygen was used in the previous runs. The final molar $\mathrm{O}_{2} / p$-xylene ratio was 6 . We had originally intended for the final molar $\mathrm{O}_{2} / p$-xylene ratio to be 9 in this experiment, but the reactor pressure was high, so we stopped adding oxygen at a ratio of 6 . The overall rate of oxygen addition in Run 4 is almost the same as that in Run 2, however, the product selectivity from Run 4 is different from that in Run 2. From Table 1 and Figure 3a, the TPA selectivity in Run 4 at 80 min was only $7.3 \mathrm{~mol} \%$ and the $p$-xylene selectivity was $87.9 \mathrm{~mol} \%$, indicating that
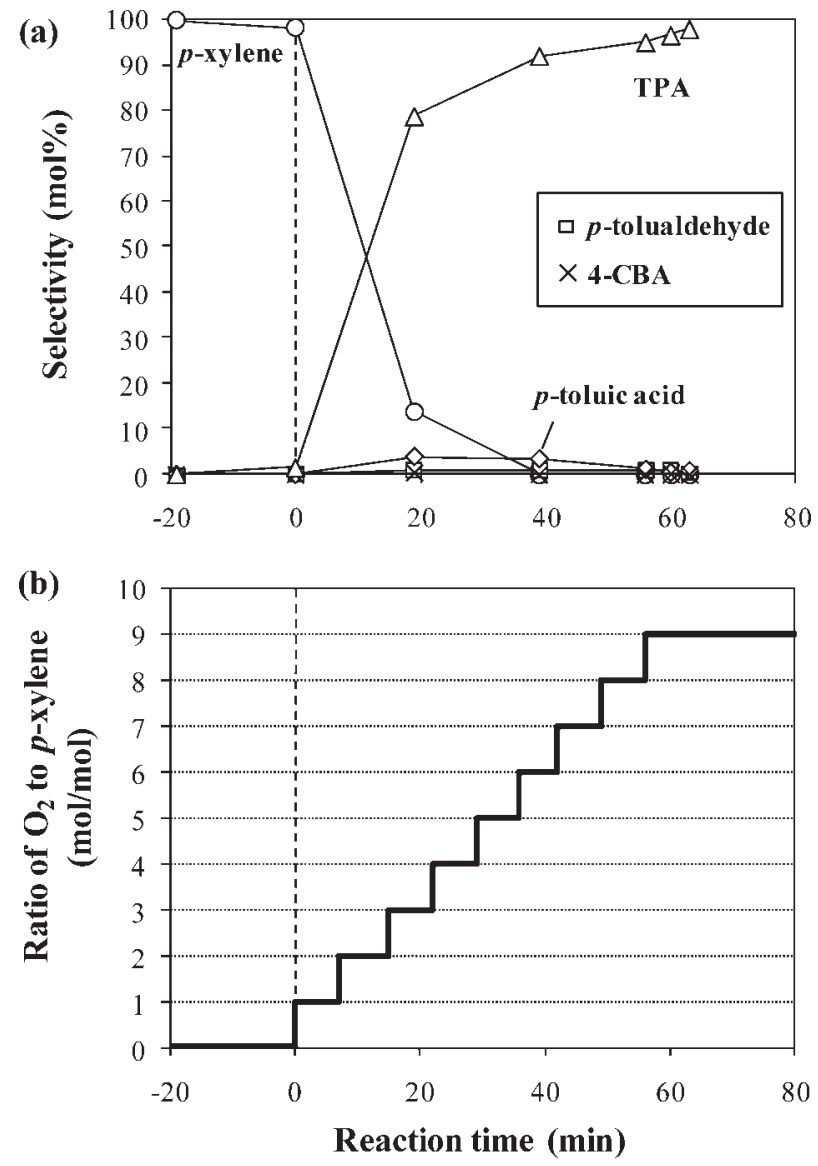

Figure 4. Results from $p$-xylene partial oxidation in HTW (Run 3).

(a) Product selectivity and (b) oxygen feed program. 
$p$-xylene partial oxidation did not proceed very quickly in this condition. On the other hand, the TPA selectivity in Run 2 at $80 \mathrm{~min}$ was $70 \mathrm{~mol} \%$ and the $p$-xylene selectivity was $0 \mathrm{~mol} \%$ as shown in Figure 2a. The final reactor pressure from Run 4 was higher than that of Run 2 even though less oxygen was added. This observation indicates that less oxygen was consumed in Run 4 and is consistent with the high $p$-xylene selectivity after the reaction in Run 4 shown in Table 1.

Run 5 is also an experiment with a continuous feed of oxygen. The overall rate of oxygen feed is faster than in Run 4 and it is almost the same as that in Run 3. Table 1 shows that the TPA selectivity from Run 5, $31.4 \mathrm{~mol} \%$, was higher than that from Run 4, but it was lower than that from Run 3, which used step-wise oxygen addition. The $p$-xylene selectivity from Run 5 was lower than Run 4, but it was higher than from Run 3. The pressure of Run 5 is lower than that of Run 4 , indicating that the amount of oxygen consumed in Run 5 is greater than that in Run 4. We also conducted another experiment with continuous oxygen feed, Run 6, but with slightly faster addition than in Run 5 . The product selectivity (Table 1) and the pressure of the batch reactor (Figure 3) from Run 6 were similar to those of Run 5. The results from Runs 4-6 indicate that continuous oxygen feed is not effective for $p$-xylene partial oxidation in HTW. Rather, rapid oxygen addition in small increments, as in Run 3, is much more effective from the view of both reaction rate and TPA selectivity.

The results above indicate a significant effect of the oxygen feed method on the course of the $p$-xylene partial oxidation reaction. However, it is possible that the large differences in the final product distributions from Runs 2-6 are due not only to the oxygen feed method, but perhaps also to differences in temperature. There was temperature difference of about $10^{\circ} \mathrm{C}$ between Run 2-6 as shown in Figure 3c, because the temperature increased from about 290 to $300^{\circ} \mathrm{C}$ when the oxygen was injected at $t=0$. To determine the significance of this temperature difference, we conducted an experiment (Run 7) in which temperature was initially set at $300^{\circ} \mathrm{C}$ and it then increased to $310^{\circ} \mathrm{C}$ when the oxygen was injected at $t=0$. The other experimental conditions of Run 7 are identical with those in Run 6. Table 1 shows no major differences in the product selectivities between Run 6 and 7 . This result indicates that the differences in the product selectivities in Runs 2-6 are not due to temperature effects. Rather, the oxygen feed method appears to be the chief influence.

\section{Discussion}

The results presented above indicate that the manner in which oxygen is added to the reactor has a profound influence on the progression of $p$-xylene oxidation in HTW. Adding oxygen quickly in intermittent small bursts (as in Runs 1-3) must have some beneficial impact on the controlling physics or chemistry. In this section, we propose a potential explanation for the oxygen addition strategy having the effect observed. This hypothesis involves both physical and chemical effects.

The rapid introduction of gaseous oxygen into the reactor provides additional turbulence and mixing to the fluid present at the end of the dip tube. In contrast, the much slower continuous addition of oxygen (as in Runs 4-7) would be much less disruptive to the local fluid environment. In addition to being mixed more vigorously, the fluid around the dip tube would also have higher local oxygen concentrations in the experiments with rapid step-wise oxygen addition. This combination of better mixing and higher local oxygen concentrations may be the factor that leads to faster reaction rates with step-wise addition of oxygen.

The literature indicates that there are two chief roles of oxygen in $p$-xylene partial oxidation in $\mathrm{HTW}^{7,14}$ One is the formation of an active catalyst species, $\mathrm{Mn}(\mathrm{II})-\left(\mathrm{Br}^{\bullet}\right)$, from $\mathrm{MnBr}_{2}$ as follows.

$$
\begin{aligned}
\mathrm{Mn}(\mathrm{II})+\mathrm{Br}^{-} & \rightarrow \mathrm{Mn}(\mathrm{II})-\mathrm{Br}^{-} \underset{\text { oxidant }}{\rightarrow} \mathrm{Mn}(\mathrm{III})-\mathrm{Br}^{-} \\
& \rightarrow \mathrm{Mn}(\mathrm{II})-\left(\mathrm{Br}^{\bullet}\right)
\end{aligned}
$$

The oxidant in the second step is typically a peroxy radical and a peroxide. The higher local oxygen concentrations in HTW could more readily form these oxidant species. The other role of oxygen is a reactant in the $p$-xylene partial oxidation reaction as follows.

$$
\begin{gathered}
\mathrm{ArCH}_{2}^{\bullet}+1.5 \mathrm{O}_{2} \rightarrow \mathrm{ArCOO}^{\bullet}+\mathrm{H}_{2} \mathrm{O} \\
\mathrm{ArCOO}^{\bullet}+\mathrm{ArCH}_{3} \rightarrow \mathrm{ArCOOH}+\mathrm{ArCH}_{2}^{\bullet}
\end{gathered}
$$

Reaction 5 is a composite reaction (not an elementary step), and it is given here along with Reaction 6 simply to show that $\mathrm{O}_{2}$ will play a role in forming peroxy radicals and peroxides, which are thought to be important in maintaining the catalyst activity, as shown in Reaction 4.

When oxygen is added continuously to the reactor, its rate of addition is much slower and perhaps slow enough to allow equilibration between the vapor and liquid phases that coexist in the reactor. If this is the case, the oxygen concentration in the liquid phase will increase continuously as more oxygen is added, but the equilibrium concentration may never be as high as the temporary, nonequilibrium concentrations that are encountered by the rapid addition of step-wise oxygen bursts.

We evaluated the equilibrium distribution of oxygen in the liquid and gas phases at $300^{\circ} \mathrm{C}$ by using a phase equilibrium correlation. ${ }^{15}$ In this calculation, we assume that only water $(270 \mathrm{~g})$ and oxygen $(0.243 \mathrm{~mol}$, which corresponds to a molar $\mathrm{O}_{2} / p$-xylene ratio of 3 ) are present, since the correlation is for this binary system and the amount of organic material present is small by comparison. The calculation indicates that about $47 \%$ of the oxygen added would be dissolved in the liquid water phase at equilibrium, and the other $53 \%$ would be in the gas phase. At $300^{\circ} \mathrm{C}$, the volumes of the liquid and gas phases in the reactor are $383 \mathrm{~mL}$ and 57 $\mathrm{mL}$, respectively. Therefore, the concentrations of oxygen in the liquid and gas phase are $0.30 \mathrm{~mol} \mathrm{~L}^{-1}$ and $2.30 \mathrm{~mol}$ $\mathrm{L}^{-1}$, respectively. For the case of step-wise oxygen feed, as in Runs 2 and 3, there may be locations with higher nonequilibrium concentrations of oxygen $\left(>0.30 \mathrm{~mol} \mathrm{~L}^{-1}\right)$ around the tube outlet in the liquid phase when the oxygen is injected. Therefore, $p$-xylene partial oxidation might proceed more readily in Runs 2 and 3 than in Runs 4-6. 


\section{Conclusions}

This work demonstrates that TPA can be produced in high-selectivity (>90 mol \%) and high-yield (>80 mol \%) via synthesis in HTW from $p$-xylene at a high initial concentration $\left(0.2 \mathrm{~mol} \mathrm{~L}^{-1}\right)$. This initial $p$-xylene concentration is the highest used to date in successful HTW synthesis for this system. We found that increasing the catalyst concentration slightly increased the rate of TPA formation, but it did not affect the final product distribution. On the other hand, we found that details about the oxygen feed method, such as feed speed and amount, significantly affected the final product distribution.

When we added oxygen slowly and continuously, a large portion of $p$-xylene remained unreacted. In contrast, when we add oxygen quickly and in small discrete increments, high selectivities of TPA were obtained. This outcome may be due to instantaneously high local oxygen concentrations in the liquid phase that may accompany this oxygen feed strategy. These results suggest that achievement of high selectivity for TPA synthesis at even higher $p$-xylene concentrations may be possible by optimizing the oxygen feed method (e.g., magnitude, duration, and frequency of oxygen bursts).

Although a tank-type reactor was used in these experiments, the results can readily be generalized to a tubular flow reactor. Here, one could obtain rapid introduction of oxygen bursts by feeding oxygen intermittently through sidestream jets along the tube length. The HTW solution with catalyst and $p$-xylene would flow continuously within the tube.

\section{Acknowledgments}

This research was supported in part by the National Science Foundation (CTS-0625641) and also by the Institute of National Colleges of Technology, Japan.

\section{Literature Cited}

1. Savage PE. Organic chemical reactions in supercritical water. Chem Rev. 1999;99:603-621.

2. Akiya N, Savage PE. Roles of water for chemical reactions in high temperature water. Chem Rev. 2002;102:2725-2750.

3. Dunn JB, Savage PE. Economic and environmental assessment of high-temperature water as a medium for terephthalic acid synthesis. Green Chem. 2003;5:649-655.

4. Fraga-Dubreuil J, Poliakoff M. Organic reactions in high-temperature and supercritical water. Pure Appl Chem. 2006;78:1971-1982.

5. Dunn JB, Savage PE. High-temperature liquid water: a viable medium for terephthalic acid synthesis. Environ Sci Technol. 2005;39: 5427-5435.

6. Dunn JB, Savage PE. Terephthalic acid synthesis in high-temperature liquid water. Ind Eng Chem Res. 2002;41:4460-4465.

7. Dunn JB, Urquhart DI, Savage PE. Terephthalic acid synthesis in supercritical water. Adv Synth Catal. 2002;344:385-392.

8. Savage PE, Dunn JB, Yu J. Recent advances in catalytic oxidation in supercritical water. Combust Sci Technol. 2006;178:443-465.

9. Hamley PA, Ilkenhans T, Webster JM, Garcia-Verdugo E, Venardou E, Clarke MJ, Auerbach R, Thomas WB, Whiston K, Poliakoff M. Selective partial oxidation in supercritical water: the continuous generation of terephthalic acid from para-xylene in high yield. Green Chem. 2002;4:235-238.

10. Garcia-Verdugo E, Venardou E, Thomas WB, Whiston K, Partenheimer W, Hamley PA, Poliakoff M. Is it possible to achieve highly selective oxidations in supercritical water? Aerobic oxidation of methylaromatic compounds. Adv Synth Catal. 2004;346:307-316.

11. Garcia-Verdugo E, Fraga-Dubreuil J, Hamley PA, Thomas WB, Whiston K, Poliakoff M. Simultaneous continuous partial oxidation of mixed xylenes in supercritical water. Green Chem. 2005;7:294-300.

12. Holliday RL, Jong BYM, Kolis JW. Organic synthesis in subcritical water oxidation of alkyl aromatics. J Supercrit Fluids. 1998;12:255-260.

13. Pryor WA, Jentoft RE. Solubility of $\mathrm{m}$ - and p-xylene in water and in aqueous ammonia from $0^{\circ} \mathrm{C}$ to $300^{\circ} \mathrm{C}$. J Chem Eng Data. 1961;6:36-37.

14. Chen Y, Fulton JL, Partenheimer W. The structure of the homogeneous oxidation catalyst, $\mathrm{Mn}(\mathrm{II})\left(\mathrm{Br}^{-1}\right)_{\mathrm{X}}$, in supercritical water: an X-ray absorption fine-structure study. J Am Chem Soc. 2005;127: 14085-14093.

15. Fernández-Prini R, Alvarez JL, Harvey AH. Henry's constants and vapor-liquid distribution constants for gaseous solutes in $\mathrm{H}_{2} \mathrm{O}$ and $\mathrm{D}_{2} \mathrm{O}$ at high temperatures. $J$ Phys Chem Ref Data. 2003;32:903-916.

Manuscript received May 30, 2008, revision received Aug. 2, 2008, and final revision received Sept. 26, 2008. 